\title{
PRIMITIVE SUBGROUPS AND PST-GROUPS
}

\author{
A. BALLESTER-BOLINCHES, J. C. BEIDLEMAN ${ }^{\bowtie}$ and R. ESTEBAN-ROMERO
}

(Received 2 May 2013; accepted 13 May 2013; first published online 18 July 2013)

\begin{abstract}
All groups considered in this paper are finite. A subgroup $H$ of a group $G$ is called a primitive subgroup if it is a proper subgroup in the intersection of all subgroups of $G$ containing $H$ as a proper subgroup. He et al. ['A note on primitive subgroups of finite groups', Commun. Korean Math. Soc. 28(1) (2013), 55-62] proved that every primitive subgroup of $G$ has index a power of a prime if and only if $G / \Phi(G)$ is a solvable PST-group. Let $\mathfrak{X}$ denote the class of groups $G$ all of whose primitive subgroups have prime power index. It is established here that a group $G$ is a solvable PST-group if and only if every subgroup of $G$ is an $\mathfrak{Z}$-group.
\end{abstract}

2010 Mathematics subject classification: primary 20D10; secondary 20D15, 20D20.

Keywords and phrases: finite groups, primitive subgroups, solvable PST-groups, $T_{0}$-groups.

\section{Introduction and statements of results}

All groups considered here are finite. A subgroup $H$ of a group $G$ is called primitive if it is a proper subgroup in the intersection of all subgroups containing $H$ as a proper subgroup. All maximal subgroups of $G$ are primitive. Some properties of primitive subgroups are given in Lemma 2.1 and include:

(a) every proper subgroup of $G$ is the intersection of a set of primitive subgroups of $G$;

(b) if $X$ is a primitive subgroup of a subgroup $T$ of $G$, then there exists a primitive subgroup $Y$ of $G$ such that $X=Y \cap T$.

Johnson [10] introduced the concept of primitive subgroup of a group. He proved that a group $G$ is supersolvable if every primitive subgroup of $G$ has prime power index in $G$.

The next results on primitive subgroups of a group $G$ indicate how such subgroups give information about the structure of $G$.

THeOREM 1.1 [7]. Let $G$ be a group. The following statements are equivalent:

(1) every primitive subgroup of $G$ containing $\phi(G)$ has prime power index;

(2) $G / \phi(G)$ is a solvable PST-group.

(C) 2013 Australian Mathematical Publishing Association Inc. 0004-9727/2013 \$16.00 
THeOREM 1.2 [6]. Let $G$ be a group. The following statements are equivalent:

(1) every primitive subgroup of $G$ has prime power index;

(2) $G=[L] M$ is a supersolvable group, where L and $M$ are nilpotent Hall subgroups of $G, L$ is the nilpotent residual of $G$ and $G=L \mathrm{~N}_{G}(L \cap X)$ for every primitive subgroup $X$ of $G$. In particular, every maximal subgroup of $L$ is normal in $G$.

Note that $G=[L] M$ in Theorem 1.2 means that $G$ is the semidirect product of $L$ by $M$.

Let $\mathfrak{X}$ denote the class of groups $G$ such that the primitive subgroups of $G$ have prime power index (see [5, pages 132-137]). By (a) it is clear that $\mathfrak{X}$ consists of those groups whose subgroups are intersections of subgroups of prime power indices.

One purpose of this paper is to characterise solvable PST-groups in terms of $\mathfrak{X}$-subgroups.

A subgroup $H$ of a group $G$ is said to be $S$-permutable in $G$ if it permutes with the Sylow subgroups of $G$. Kegel proved that an $S$-permutable subgroup of $G$ is subnormal in $G$ (see [2, Theorem 1.2.14]). $S$-permutability is said to be transitive in $G$ if, whenever $H$ and $K$ are subgroups of $G$ such that $H$ is $S$-permutable in $K$ and $K$ is $S$-permutable in $G$, then $H$ is $S$-permutable in $G$. A group $G$ is said to be a PST-group if $S$-permutability is a transitive relation in $G$. By Kegel's result, $G$ is a PST-group if and only if every subnormal subgroup of $G$ is $S$-permutable. Agrawal [1] characterised solvable PST-groups. He proved the following theorem.

THeOREM 1.3. Let $G$ be a solvable group. $G$ is a PST-group if and only if it has an abelian normal Hall subgroup $N$ such that $G / N$ is nilpotent and $G$ acts by conjugation on $N$ as a group of power automorphisms.

In Theorem 1.3, $N$ can be taken to be the nilpotent residual of $G$. From Theorem 1.3 it follows that subgroups of solvable PST-groups are solvable PST-groups. Many interesting results about PST-groups can be found in [2, Ch. 2].

THEOREM A. Let G be a group. The following statements are equivalent:

(1) $G$ is a solvable PST-group;

(2) every subgroup of $G$ is an $\mathfrak{X}$-group.

Let $G$ be an $\mathfrak{X}$-group. It follows from Theorem A that if $G$ is not a solvable PSTgroup, then $G$ has a subgroup $H$ which does not belong to $\mathfrak{X}$. See Examples 4.1 and 4.2.

A well-known theorem of Lagrange (see [13, Ch. 1, Theorem 1.3.6]) states that given a subgroup $H$ of a group $G$, the order of $G$ is the product of the order $|H|$ of $H$ and the index $|G: H|$ of $H$ in $G$. In particular, the order of any subgroup divides the order of the group. The converse, namely, if $d$ divides the order of a group $G$, then $G$ has a subgroup of order $d$, is not true in general. Groups satisfying this condition are often called CLT-groups. The alternating group of order 12, having no subgroups of order six, is an example of a non-CLT-group.

On the other hand, abelian groups contain subgroups of every possible order, and it is not difficult to prove that a group is nilpotent if and only if it contains a normal 
subgroup of each possible order [8]. Ore [11] and Zappa [15] obtained a similar characterisation for supersolvable groups. Further results on supersolvable groups can be found in [3].

THeOREM 1.4. A group $G$ is supersolvable if and only if each subgroup $H \leq G$ contains a subgroup of order $d$ for each divisor $d$ of $|H|$.

Of course, we can state Theorem 1.4 in the following equivalent way, more easily treated.

THEOREM 1.5. A group $G$ is supersolvable if and only if each subgroup $H \leq G$ contains a subgroup of index $p$ for each prime divisor $p$ of $|H|$.

A proof of this theorem can be found in [5, Ch. 1, Theorem 4.2]. It must be noted that CLT-groups are not necessarily supersolvable, as the symmetric group of order four shows.

The condition on a group $G$ given in Theorem 1.5 , namely,

for all $H \leq G$ and for all primes $q$ dividing $|H|$, there exists a subgroup $K$ of $G$ such that $K \leq H$ and $|H: K|=q$,

has a dual formulation:

for all $H \leq G$ and for all primes $q$ dividing $|G: H|$, there exists a subgroup $K$ of $G$ such that $H \leq K$ and $|K: H|=q$.

Groups satisfying the latter condition have been studied by some authors. Following [5, Ch. 1, Section 4], we will call them $\mathcal{Y}$-groups.

A group $G$ is said to be a $\mathcal{Y}$-group if for all subgroups $H$ of $G$ and all primes $q$ dividing the index $|G: H|$ of $H$ in $G$, there exists a subgroup $K$ of $G$ with $H \leq K$ and $|K: H|=q$.

Note that a group $G$ is a $\mathcal{Y}$-group if and only if for every subgroup $H$ of $G$ and for every natural number $d$ dividing $|G: H|$ there exists a subgroup $K$ of $G$ such that $H \leq K$ and $|K: H|=d$. The following characterisation of $\mathcal{Y}$-groups appears in [5, Ch. 1, Theorem 4.3].

Theorem 1.6. Let $L=G^{\mathfrak{N}}$ be the nilpotent residual of the group $G$. Then $G$ is a $\mathcal{Y}$ group if and only if $L$ is a nilpotent Hall subgroup of $G$ such that for all subgroups $H$ of $L, G=L \mathrm{~N}_{G}(H)$.

From Theorem 1.6, we see that if $G \in \mathcal{Y}$ and $X$ is a normal subgroup of $L$, then $X$ is normal in $G$. In particular, $\mathcal{Y}$-groups are supersolvable. Moreover, if $G \in \mathcal{Y}$, then $L$ must have odd order.

Further results on $\mathcal{Y}$-groups can be found in [5, Ch. 4, Theorems 5.2 and 5.3]. For example, a solvable group $G$ is a $\mathcal{Y}$-group if and only if every subgroup of $G$ can be written as an intersection of subgroups of $G$ of coprime prime power indices.

From Theorems 1.3 and 1.6 we obtain the following theorem. 
Theorem 1.7. Let $G$ be a $\mathcal{Y}$-group with nilpotent residual $L$.

(1) $G$ is a solvable PST-group if and only if $L$ is abelian.

(2) $G / \phi(G)$ is a solvable PST-group.

We note that the class $\mathcal{Y}$ is a subclass of the class $\mathfrak{X}$ by Theorems 1.2 and 1.7. The example of Humphreys in [5, page 136] (see also [9]) shows that $\mathcal{Y}$ is a proper subclass of $\mathfrak{X}$.

THeOREM B. Let G be a group. The following statements are equivalent:

(1) $G$ is a solvable PST-group;

(2) every subgroup of $G$ is a $\mathcal{Y}$-group;

(3) every subgroup of $G$ is an $\mathfrak{X}$-group.

Let $\mathfrak{F}$ be a class of groups. Denote by $\mathcal{S} \mathfrak{F}$ (respectively, $\mathcal{S}(\mathfrak{F})$ ) the class of groups all of whose subgroups are $\mathfrak{F}$-groups (respectively, solvable $\mathfrak{F}$-groups).

Theorem C. We have

$$
\mathcal{S} \mathfrak{X}=\mathcal{S} Y=S T_{0}=\mathcal{S}\left(T_{0}\right)=\mathcal{S P S T}=\mathcal{S}(\mathrm{PST})=\mathcal{S}\left(\mathrm{PST}_{0}\right)=\mathcal{S}\left(P T_{0}\right) .
$$

We mention that $\mathcal{S} \mathfrak{X}=\mathcal{S} \mathcal{Y}$ of Theorem $\mathrm{C}$ follows from Theorem B and is [5, Theorem 5.3, page 135]. The proof of [5, Theorem 5.3] is very different and more difficult than the proof of Theorem B.

\section{Preliminaries}

Lemma $2.1[6,7,10]$. Let $G$ be a group. The following statements hold.

(1) For every proper subgroup $H$ of $G$, there is a set of primitive subgroups $\left\{X_{i} \mid i \in I\right\}$ in $G$ such that $H=\bigcap_{i \in I} X_{i}$.

(2) If $H \leq G$ and $T$ is a primitive subgroup of $H$, then $T=H \cap X$ for some primitive subgroup $X$ of $G$.

(3) If $K \unlhd G$ and $K \leq H \leq G$, then $H$ is a primitive subgroup of $G$ if and only if $H / K$ is a primitive subgroup of $G / K$.

(4) Let $P$ and $Q$ be subgroups of $G$ with $(|P|,|Q|)=1$. Suppose that $H$ is a subgroup of $G$ such that $H P \leq G$ and $H Q \leq G$. Then $H P \cap H Q=H$. In particular, if $H$ is a primitive subgroup of $G$, then $P \leq H$ or $Q \leq H$.

Let $G$ be a group. We call $G$ a T-(respectively, PT-)group if $H \unlhd K \unlhd G$ (respectively, $H$ is permutable in $K$ and $K$ is permutable in $G$ ) implies $H \triangleleft G$ (respectively, $H$ is permutable in $G$ ). By Kegel's result, $G$ is a PT-group if and only if every subnormal subgroup of $G$ is permutable. Many results about T- and PT-groups can be found in [2, Ch. 2]. We call $G$ a $\mathrm{T}_{0}$-group if $G / \phi(G)$ is a T-group, where $\phi(G)$ is the Frattini subgroup of $G$. $\mathrm{T}_{0}$-groups have been studied in $[4,12,14]$. Several of the results on $\mathrm{T}_{0}$-groups given in $[4,12]$ are contained in the next three lemmas and are needed in the proof of Theorem A.

A group $G$ is called a $\mathrm{PT}_{0^{-}}$(respectively, $\mathrm{PST}_{0^{-}}$)group provided that $G / \phi(G)$ is a PT-(respectively, PST-)group. For solvable groups we have the following lemmas. 
Lemma 2.2 [12]. We have $\mathcal{S}\left(T_{0}\right)=\mathcal{S}\left(P T_{0}\right)=\mathcal{S}\left(\mathrm{PST}_{0}\right)$.

Lemma 2.3 [4]. Let $G$ be a group. Then $G$ is a solvable PST-group if and only if every subgroup of $G$ is a $T_{0}$-group.

\section{Proofs of the theorems}

Proof of Theorem A. Let $G$ be a solvable PST-group and let $L$ be the nilpotent residual of $G$. By Theorem 1.3, $L$ is a normal abelian Hall subgroup of $G$ on which $G$ acts by conjugation as a group of power automorphisms. Let $X$ be a subgroup of $L$. Since $X \triangleleft G, G=L \mathrm{~N}_{G}(X)$. Let $D$ be a system normaliser of $G$. By [13, Theorem 9.2.7, page 264], $G=[L] D$, the semidirect product of $L$ by $D$. It follows by Theorem 1.2 that every primitive subgroup of $G$ has prime power index, and hence $G$ is an $\mathfrak{X}$ group. Since every subgroup of $G$ is a solvable PST-group, every subgroup of $G$ is an $\mathfrak{X}$-group.

Conversely, assume that every subgroup of $G$ is an $\mathfrak{X}$-group. We are to show that $G$ is a solvable PST-group. Let $H$ be a subgroup of $G$. Because of Theorem 1.1, $H / \phi(H)$ is a solvable PST-group, and hence $H$ is a solvable PST $_{0}$-group. By Lemma 2.2, $H$ is a $\mathrm{T}_{0}$-group. It follows that every subgroup of $G$ is a solvable $\mathrm{T}_{0}$-group and by Lemma 2.3, $G$ is a solvable PST-group.

This completes the proof.

Proof of Theorem B. Let $G$ be a solvable PST-group. Using the proof of the first part of Theorems A and 1.6, we see that every subgroup of $G$ is a $\mathcal{Y}$-group and (1) implies (2). Since $\mathcal{Y} \subseteq \mathfrak{X},(2)$ implies (3). By Theorem A we see that (3) implies (1).

Proof of Theorem C. By Theorem B, $\mathcal{S} \mathfrak{X}=\mathcal{S} \mathcal{Y}=\mathcal{S}(\mathrm{PST})=\mathcal{S P S T}$. Note, by Theorem 1.1, $\mathcal{S}\left(\mathrm{T}_{0}\right)=\mathcal{S} \mathrm{T}_{0}=\mathcal{S} \mathfrak{X}$. Finally, it follows that $\mathcal{S}\left(\mathrm{T}_{0}\right)=\mathcal{S}\left(\mathrm{PST}_{0}\right)=\mathcal{S}\left(\mathrm{PT}_{0}\right)$ by Lemma 2.2. Hence Theorem $\mathrm{C}$ holds.

\section{Examples}

ExAmple 4.1. Let $P=\left\langle x, y \mid x^{5}=y^{5}=[x, y]^{5}=1\right\rangle$ be an extra-special group of order 125 of exponent 5. Let $z=[x, y]$ and note $Z(P)=\Phi(P)=\langle z\rangle$. Then $P$ has an automorphism $a$ of order four given by $x^{a}=x^{2}, y^{a}=y^{2}$ and $z^{a}=z^{4}=z^{-1}$. Put $G=[P]\langle a\rangle$ and note $Z(G)=1, \Phi(G)=\langle z\rangle$ and $G / \Phi(G)$ is a T-group. Thus $G$ is a solvable $\mathrm{T}_{0}$-group. Let $H=\langle y, z, a\rangle$ and notice $\Phi(H)=1$. Then $H$ is not a T-group since the nilpotent residual $L$ of $H$ is $\langle y, z\rangle$ and $a$ does not act on $L$ as a power automorphism. Thus $H$ is not a $\mathrm{T}_{0}$-group, and hence not a solvable PST-group. By Theorem 1.1, $G$ is an $\mathfrak{X}$-group and $H$ is not an $\mathfrak{X}$-group.

ExAmple 4.2. Let $P=\left\langle x, y \mid x^{3}=y^{3}=[x, y]^{3}=1\right\rangle$ be an extra-special group of order $3^{3}$ and exponent 3. Then $P$ has an automorphism $b$ of order two given by $x^{b}=x^{-1}$, $y^{b}=y^{-1}$ and $[x, y]^{b}=1$. Let $G=[P]\langle b\rangle$ and note $Z(G)=Z(P)=\langle[x, y]\rangle=\phi(G)$. Then $G / \phi(G)$ is a T-group, and hence $G$ is a $\mathrm{T}_{0}$-group. By Lemma 2.3, $G$ has a subgroup which is not a $\mathrm{T}_{0}$-group, and hence not a solvable PST-group. Note that $G$ is an $\mathfrak{X}$-group. 


\section{References}

[1] R. K. Agrawal, 'Finite groups whose subnormal subgroups permute with all Sylow subgroups', Proc. Amer. Math. Soc. 47 (1975), 77-83.

[2] M. Asaad, A. Ballester-Bolinches and R. Esteban-Romero, Products of Finite Groups, de Gruyter Expositions in Mathematics, 53 (Walter de Gruyter, Berlin, 2010).

[3] A. Ballester-Bolinches, J. C. Beidleman and R. Esteban-Romero, 'On some classes of supersoluble groups', J. Algebra 312(1) (2007), 445-454.

[4] A. Ballester-Bolinches, R. Esteban-Romero and M. C. Pedraza-Aguilera, 'On a class of $p$-soluble groups', Algebra Colloq. 12(2) (2005), 263-267.

[5] H. G. Bray, W. E. Deskins, D. Johnson, J. F. Humphreys, B. M. Puttaswamaiah, P. Venzke and G. L. Walls, Between Nilpotent and Solvable (ed. M. Weinstein) (Polygonal, Washington, NJ, 1982).

[6] W. Guo, K. P. Shum and A. Skiba, 'On primitive subgroups of finite groups', Indian J. Pure Appl. Math. 37(6) (2006), 369-376.

[7] X. He, S. Qiao and Y. Wang, 'A note on primitive subgroups of finite groups', Commun. Korean Math. Soc. 28(1) (2013), 55-62.

[8] C. V. Holmes, 'Classroom notes: a characterization of finite nilpotent groups', Amer. Math. Monthly 73(10) (1966), 1113-1114.

[9] J. F. Humphreys, 'On groups satisfying the converse of Lagrange's theorem', Proc. Cambridge Philos. Soc. 75 (1974), 25-32.

[10] D. L. Johnson, 'A note on supersoluble groups', Canad. J. Math. 23 (1971), 562-564.

[11] O. Ore, 'Contributions to the theory of groups of finite order', Duke Math. J. 5(2) (1939), 431-460.

[12] M. F. Ragland, 'Generalizations of groups in which normality is transitive', Comm. Algebra 35(10) (2007), 3242-3252.

[13] D. J. S. Robinson, A Course in the Theory of Groups, 2nd edn, Graduate Texts in Mathematics, 80 (Springer, New York, 1996).

[14] R. W. van der Waall and A. Fransman, 'On products of groups for which normality is a transitive relation on their Frattini factor groups', Quaest. Math. 19(1-2) (1996), 59-82.

[15] G. Zappa, 'Remark on a recent paper of O. Ore', Duke Math. J. 6 (1940), 511-512.

\section{A. BALLESTER-BOLINCHES, Departament d'Àlgebra, Universitat de València, Dr. Moliner 50, 46100 Burjassot, València, Spain e-mail: adolfo.ballester@uv.es}

J. C. BEIDLEMAN, Department of Mathematics, University of Kentucky, Lexington, KY 40506-0027, USA e-mail: clark@ms.uky.edu

R. ESTEBAN-ROMERO, Departament d'Àlgebra, Universitat de València, Dr. Moliner 50, 46100 Burjassot, València, Spain e-mail: resteban@uv.es 